\title{
Clinical guidelines: what can we do to increase their use?
}

\author{
Richard Grol and Heather Buchan
}

\section{Strategies to close the gap between development and implementation of guidelines}

工 $\mathrm{n}$ the past decade, evidence-based clinical guidelines have become a major feature of health care. Researchers and clinicians in many countries have established programs to summarise the evidence for managing specific health problems and to disseminate practice guidelines.

However, clinical use of guideline recommendations does not necessarily follow. Numerous studies show that recommendations are frequently not applied in practice and that many patients do not profit from evidence-based insights. ${ }^{1}$ Large variations in performance between clinicians, practices and institutions are commonly observed.

Two reports in this issue of the Journal on pages 305 and 310 illustrate this well. ${ }^{2,3}$ In the first, an audit in a hospital outpatient clinic showed that large numbers of patients with diabetes do not achieve recommended treatment targets for control of glycaemia, blood pressure and lipid levels, despite evidence that control of these risk factors produces better outcomes. ${ }^{2}$ The second, a study of six Australian dialysis units, showed that, despite high levels of awareness of iron guideline recommendations in participating units, there is considerable variation in achievement of targets and widely differing practices in unit processes for iron management. ${ }^{3}$

Guidelines are expensive - the cost of producing a single guideline may range from US\$50 to US\$500 000, not to mention the substantial donated time from many contributors. ${ }^{4}$ Given the lack of practical impact of many clinical guidelines, a critical observer might well ask, "Why spend so much money and effort on something that is so poorly adhered to in practice?" However, the question should really be, "What can we do to increase the use of best evidence guideline recommendations?"

Guideline developers, research funders, health care managers and policymakers may need to consider a few key strategies:

- the need for greater focus on producing guidelines in formats that promote their use;

- the requirement for planned (and funded) implementation programs that take into account the complexity of change in health care; and

- the need to improve our knowledge about cost-effective methods of achieving sustained practice changes.

Worldwide, many guideline developers give little consideration to the use of their products in the real world. The reality is that guidelines are too often "lost in translation". 5 Many current programs for guideline development seem to be "science-driven", rather than scientifically based but "customer-driven". Guideline developers would do a far better job if they focused on the needs of the end user and provided clear statements, decision aids, patient education materials and practical tools to manage difficult problems in practice. More guidelines need to identify specific evidence-based indicators and criteria for clinical performance (as the guidelines discussed in this issue of the Journal do). These provide the capacity to monitor performance and give feedback to clinicians. Public reporting of significant aspects of care quality would help meet the urgent need in society for more transparency about important aspects of health care provision, and would provide a clear imperative to improve implementation and ensure guideline recommendations are feasible and do not become outdated.

There is also a need to seek a better balance between the resources devoted to summarising evidence and developing guidelines and those spent on finding the most effective ways to improve patient care. Evidence-based guideline development reflects just one specific approach to improving care - it assumes that professionals are rational decisionmakers who will act on convincing information about the pros and cons of specific routines. Most of the time, effective change in health care demands other models, ranging from those that emphasise patient involvement in decision making to those that focus on organisational development. ${ }^{6}$ Sadly, good evidence for the costeffectiveness of many of these strategies aiming to change practice is lacking. ${ }^{6-8}$ Greater investment by research funders in studies that would improve this knowledge base would help direct implementation resources and effort to where they could be of most use.

Policymakers who seek to change health care practices need to understand that some current expectations about the impact of clinical guidelines are unrealistic. A belief that developing and disseminating systematic reviews and guidelines will improve patient care ignores the complexity of change in health care. Guidelines do not implement themselves - most need well developed, well executed and sustained implementation programs, ${ }^{7}$ and even such programs usually have only a moderate effect on performance in terms of care improvement (around 8\%-10\%). ${ }^{8}$ Many factors play crucial roles in blocking or stimulating relevant changes in performance. These are not only related to professional decision making, but also to patient behaviour, interaction with colleagues, team functioning, organisational conditions for change, resources, and economic or legal conditions. ${ }^{7,9,10}$ This aspect was clearly demonstrated in the renal impairment study. ${ }^{3}$ For most complex changes in health care, we need to build bridges between the different approaches to better care - guidelines, performance indicators and feedback; patient empowerment; quality management; organisational change; improving culture, teamwork and leadership in the workplace; and creating the necessary financial incentives. ${ }^{6}$

So, with a change of focus in current guideline development and more realistic expectations of the role of guidelines in improving patient care, with better knowledge about costs and effects of change strategies, and with clinical guidelines embedded in comprehensive programs for change, evidence-based guidelines for clinical practice may become more relevant in the future. 


\section{EDITORIALS}

\section{Author details}

Richard Grol, PhD, Director ${ }^{1}$

Heather Buchan, MB ChB, MSc, FAFPHM, Chief Executive Officer ${ }^{2}$

1 Centre for Quality of Care Research (WOK), Nijmegen,

The Netherlands.

2 National Institute of Clinical Studies, Melbourne, VIC.

Correspondence: r.grol@wok.umcn.nl

\section{References}

1 McGlynn EA, Asch SM, Adams J, et al. The quality of health care delivered to adults in the United States. N Engl J Med 2003; 348: 2635-2645.

2 Bryant W, Greenfield JR, Chisholm DJ, Campbell LV. Diabetes guidelines: easier to preach than to practise? A retrospective audit of outpatient management of type 1 and type 2 diabetes mellitus. Med J Aust 2006; 185: 305-309.

3 Irving MJ, Craig JC, Gallagher M, et al. Implementing iron management clinical practice guidelines in patients with chronic kidney disease having dialysis. Med J Aust 2006; 185: 310-314.
4 Burgers JS. Quality of clinical practice guidelines [PhD thesis]. Nijmegen: Nijmegen University, 2002.

5 Lenfant $\mathrm{C}$. Shattuck lecture: clinical research to clinical practice - lost in translation? N Engl J Med 2003; 349: 868-874.

6 Grol R. Improving the quality of medical care. Building bridges among professional pride, payer profit, and patient satisfaction. JAMA 2001; 286: 2578-2584.

7 Grol R, Wensing M, Eccles M. Improving patient care: the implementation of change in clinical practice. Oxford: Elsevier, 2004.

8 Grimshaw JM, Thomas RE, MacLennan G, et al. Effectiveness and efficiency of guideline dissemination and implementation strategies. Health Technol Assess 2004; 8 (6): iii-iv, 1-72.

9 Cabana MD, Rand CS, Powe NR, et al. Why don't physicians follow clinical practice guidelines? A framework for improvement. JAMA 1999; 282: 1458-1465.

10 Solberg L, Brekke M, Fazio J, et al. Lessons from experienced guideline implementers: attend to many factors and use multiple strategies. Jt Comm J Qual Improv 2000; 26: 171-188. 